\title{
EFICIÊNCIA DE COLETA DE PARTÍCULAS PM 10 EM UM LAVADOR VENTURI DE SEÇÃO RETANGULAR
}

\author{
A. E. DE OLIVEIRA ${ }^{1}$, V. G. G. BÉTTEGA ${ }^{1}$ \\ ${ }^{1}$ Universidade Federal de São Carlos, Departamento de Engenharia Química \\ E-mail para contato: alessandroestarque@gmail.com
}

\begin{abstract}
RESUMO - O presente trabalho objetivou avaliar a eficiência fracionária de coleta de particulado em um lavador Venturi de geometria retangular a diferentes vazões de líquido de injeção. Foram utilizadas duas amostras de material particulado de rocha fosfática de diferentes granulometrias. Os resultados obtidos indicaram aumento da eficiência de captura de particulado com o aumento da vazão de líquido. Verificou-se que, abaixo de $10 \mu \mathrm{m}$, a eficiência do equipamento reduziu-se com a diminuição do tamanho de partícula. Os dados experimentais também foram comparados com modelos de eficiência obtidos da literatura.
\end{abstract}

\section{INTRODUÇÃO}

De acordo com a U.S. Environmental Protection Agency (EPA, 2016), material particulado é uma mistura de partículas em estado sólido e gotículas líquidas encontradas no ar e que possuem variados tamanhos e composições químicas. Além dos efeitos à saúde, provocam efeitos ao meio ambiente tais como a formação de neblina, acidificação de lagos e corpos d'água, desequilíbrio de nutrientes em bacias hidrográficas e regiões costeiras, prejuízos à agricultura e a diversos ecossistemas. Neste ínterim, o termo $\mathrm{PM}_{10}$ representa a faixa de partículas que adentram o trato respiratório, sendo dividida entre partículas grosseiras (aquelas compreendidas entre 2,5 e $10 \mu \mathrm{m}$ ) e partículas finas (cujo diâmetro está abaixo de $2,5 \mu \mathrm{m}$ ), sendo que ambas as faixas estão relacionadas a efeitos observáveis na saúde humana em ambientes urbanos (WHO, 2006).

Os lavadores Venturi estão entre os mais promissores dentre os equipamentos de controle de particulados a nível industrial: coletam partículas de uma ampla faixa granulométrica com $100 \%$ de eficiência. Operam a partir da pulverização pneumática de um líquido de injeção, usualmente água, cujas gotículas formadas oferecem extensa superfície de contato com o particulado presente na corrente gasosa. A partir da ocorrência de diferentes mecanismos de captura, dentre os quais a impactação inercial e a interceptação, o particulado é coletado pelas gotículas. Alta queda de pressão está associada ao processo devido à energia disponibilizada pelo gás para a atomização das gotas de líquido (HESKETH, 1996).

Modelos de predição da eficiência do lavador Venturi são encontrados na literatura, como o de Calvert et al. (1977 apud COOPER; ALLEY, 2002), que considera a impactação inercial como principal mecanismo de coleta de particulados, como exibido na Equação 1:

$$
\eta=1-\exp \left[\frac{Q_{L} \cdot u_{G} \cdot \rho_{L} \cdot \overline{D_{x}}}{55 \cdot Q_{G} \cdot \mu_{G} \cdot \psi} \cdot\left[-0,7-2 \cdot \psi \cdot f+1,4 \cdot \ln \left(\frac{2 \cdot \psi \cdot f+0,7}{0,7}\right)+\left(\frac{0,49}{0,7+2 \cdot \psi \cdot f}\right)\right]\right]
$$


em que $f$ é um fator empírico, sendo igual a 0,25 para partículas hidrofóbicas e igual a 0,50 para partículas hidrofílicas; $\psi$ é o parâmetro de impactação inercial que deve ser obtido a partir da velocidade do gás na entrada da garganta do lavador (constrição da seção reta do equipamento onde a atomização do líquido e a captura de particulados essencialmente ocorre). O parâmetro de dimensão da gota, $\overline{D_{x}}$, deve necessariamente ser o diâmetro de Sauter.

Em seu trabalho, Ribeiro (2005) propôs uma modificação da Equação 1, relacionando o parâmetro $f$ ao comprimento da garganta do lavador, segundo a Equação 2:

$$
f=0,5161 \cdot l_{t}^{0,3005}
$$

aqui, o comprimento da garganta, $l_{t}$, é dado em $\mathrm{cm}$.

Leith e Cooper (1980, apud COSTA et al., 2004) deduziram uma expressão para a eficiência teórica máxima segundo a teoria de penetração de Calvert, conforme Equação 3:

$$
\eta=1-\exp \left[-0,124 \cdot\left(\frac{f^{2}}{\beta}\right) \cdot \frac{C \cdot \rho_{p} \cdot d_{p}^{2} \cdot \Delta P}{18 \cdot \mu_{G}{ }^{2}}\right]
$$

sendo $\Delta P$ a queda de pressão na garganta do lavador, $\beta$ o fator de correção para $\Delta P$ (adotado como 0,85$)$ e $f$ o fator empírico da Equação 1.

Portanto, os objetivos do trabalho foram: investigar a performance de um lavador Venturi na captura de partículas de amostras com diferentes granulometrias, sob diferentes vazões de líquido; avaliar a eficiência de coleta fracionária deste lavador dando ênfase às partículas na faixa $\mathrm{PM}_{10}$; comparar os dados obtidos com modelos de eficiência encontrados na literatura.

\section{MATERIAIS E MÉTODOS}

O aparato experimental utilizado incluía um lavador Venturi de seção transversal retangular, um ciclone, um soprador, uma bomba helicoidal e rotâmetro. A Figura 1 mostra um esquema representativo do aparato experimental e a Tabela 1 apresenta as principais dimensões do lavador.

Figura 1 - Esquema do lavador Venturi.

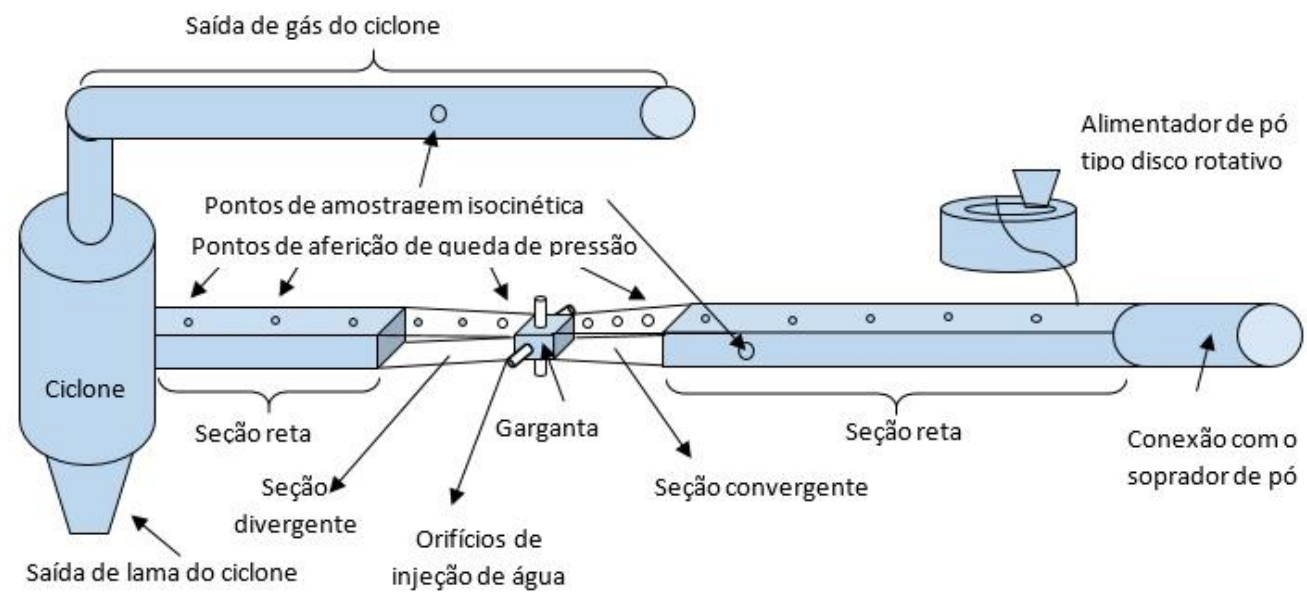

Fonte: Acervo pessoal 
Tabela 1 - Configuração geométrica do lavador Venturi

\begin{tabular}{|c|c|}
\hline Grandeza & Valor \\
\hline \hline Altura & $40 \mathrm{~mm}$ \\
\hline Largura na entrada e saída & $75 \mathrm{~mm}$ \\
\hline Largura da garganta & $27 \mathrm{~mm}$ \\
\hline Comprimento da seção convergente & $130 \mathrm{~mm}$ \\
\hline Comprimento da garganta & $120 \mathrm{~mm}$ \\
\hline Comprimento da seção divergente & $340 \mathrm{~mm}$ \\
\hline Meio ângulo convergente & $10,50^{\circ}$ \\
\hline Meio ângulo divergente & $4,00^{\circ}$ \\
\hline
\end{tabular}

Fonte: Acervo pessoal

Os testes de eficiência do lavador Venturi foram realizados com vazões de líquido de 0,3 e 0,6 L/min, sendo que a injeção de líquido era feita por um único orifício de injeção na face superior da garganta. A eficiência de coleta do lavador Venturi foi quantificada através da amostragem isocinética nos orifícios anterior e posterior à garganta, como exibido na Figura 1. Esse método consistia na retenção do material particulado em uma membrana de filtração constituída de ésteres de celulose e que possuía $0,20 \mu \mathrm{m}$ de poro, $47 \mathrm{~mm}$ de diâmetro e $150 \mu \mathrm{m}$ de espessura, $75 \%$ de porosidade e era hidrofílica. Foram utilizadas amostras de particulado de dois diferentes diâmetros volumétricos medianos de partícula, 28,3 $\mu \mathrm{m}$ (A) e 2,73 $\mu \mathrm{m}$ (B). Suas curvas granulométricas estão exibidas na Figura 2. O gás carreador era ar atmosférico e o líquido de injeção era água, ambos à temperatura ambiente. $\mathrm{O}$ material particulado utilizado era rocha fosfática $\left(\rho p=2900 \mathrm{~kg} / \mathrm{m}^{3}\right)$, também sob pressão e temperatura ambiente (aproximadamente $25^{\circ} \mathrm{C}$ e $690 \mathrm{mmHg}$ ). A velocidade da corrente gasosa na garganta foi fixada em 60,6 m/s. Para cada amostra, os testes foram feitos em duplicata.

Figura 2 - Granulometria das amostras A e B.

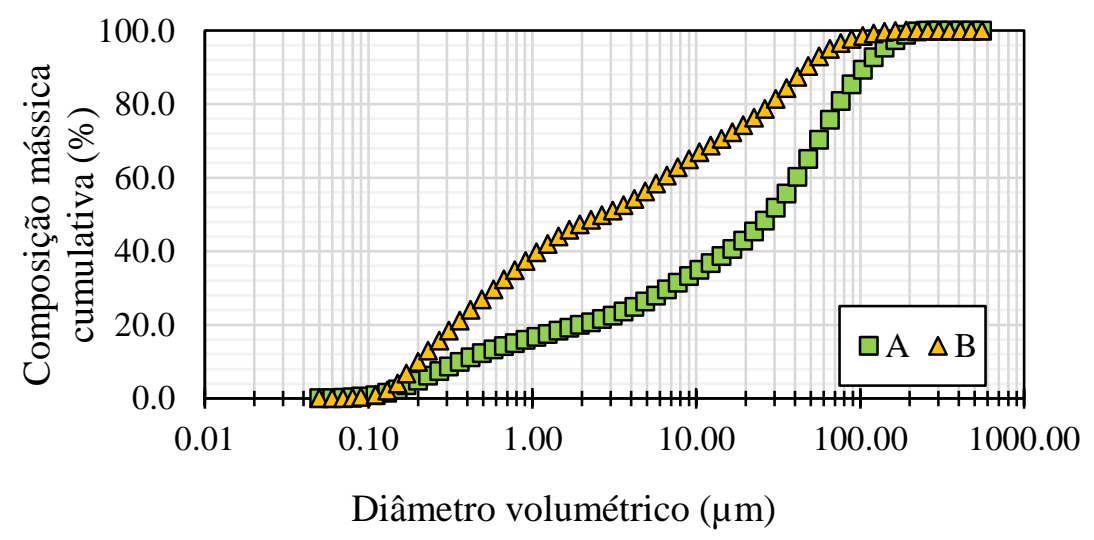

\section{RESULTADOS E DISCUSSÃO}

A Figura 3 apresenta os dados obtidos de eficiência fracionária de cada amostra sob as diferentes condições de vazão de líquido utilizadas. 


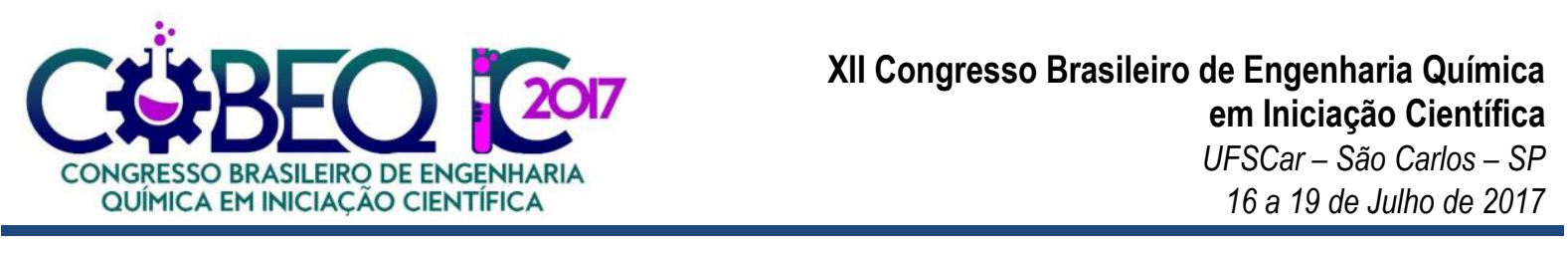

Figura 3 - Eficiência fracionária das amostras A e B.

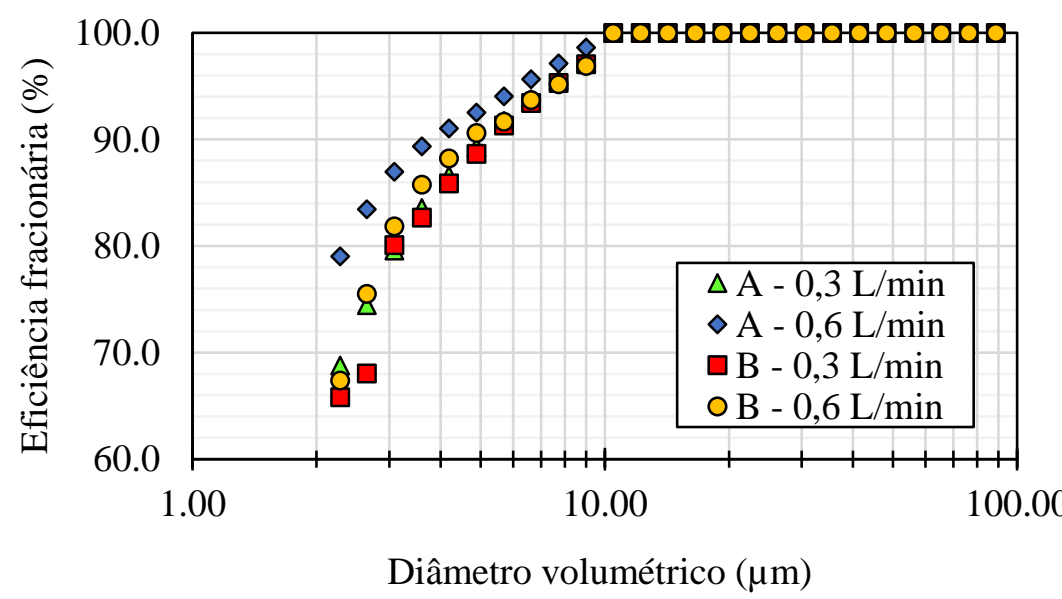

Da Figura 3, verifica-se que o processo obteve $100 \%$ de eficiência a partir de $10 \mu \mathrm{m}$ para ambas as amostras e, abaixo deste valor, ou seja, na faixa $\mathrm{PM}_{10}$, a eficiência aumentou com o aumento do diâmetro do particulado para as amostras A e B. Isto deveu-se a partículas maiores e de maior massa possuírem maior probabilidade de serem capturadas através dos mecanismos de interceptação direta e impactação inercial, respectivamente. Assumindo-se partículas esféricas, quanto maior seu raio, maior a probabilidade de que esta dimensão seja próxima da distância entre as fronteiras do particulado e as fronteiras da gota de líquido, aumentando-se a eficiência de interceptação. Ademais, o aumento da massa da partícula e sua inércia ocasiona menor probabilidade de a partícula seguir as linhas de corrente de gás quando este contorna a gota, proporcionando a colisão entre partícula e gota e aumentando-se a eficiência do mecanismo inercial. Estes mecanismos, portanto, possuem eficiências reduzidas quanto menor o tamanho da partícula, resultando em menor eficiência do processo para partículas na faixa $\mathrm{PM}_{10}$. Isto resulta em um gás emitido à atmosfera com particulados de alta periculosidade à saúde humana. Contudo, das curvas obtidas, nota-se que o aumento da vazão de líquido, a uma vazão fixa de gás, ocasionou aumento da eficiência para ambas as amostras na faixa $\mathrm{PM}_{10}$. Neste caso, houve aumento do número de gotículas de água disponíveis para uma mesma vazão de gás e concentração de particulado, resultando em maior probabilidade de as partículas sólidas serem capturadas.

Sobre a diferença nas curvas de distribuição para cada amostra, a amostra A, de diâmetro mediano igual a $28,3 \mu \mathrm{m}$, possuiu maiores eficiências que a amostra B, de diâmetro mediano igual a $2,73 \mu \mathrm{m}$, comparando-se sob as mesmas vazões de líquido. Embora esta diferença não seja evidente nas curvas de eficiência fracionária, em termos de eficiência global (ponderando-se as eficiências fracionárias pela porcentagem de partículas de cada diâmetro na amostra inicial), verifica-se que a amostra com partículas maiores (A) é mais eficientemente coletada pelo lavador que aquela constituída por partículas menores (B) devido ao aumento da eficiência dos mecanismos de coleta como anteriormente discutido. Por exemplo, para a vazão de $0,3 \mathrm{~L} / \mathrm{min}$, a eficiência global experimental foi igual a 91,81 \% para A frente a 87,66\% de B.

Os resultados experimentais (para vazão de líquido de $0,3 \mathrm{~L} / \mathrm{min}$ ) também foram comparados com equações de predição de eficiência de lavadores Venturi obtidas da literatura (Equações 1, 2 e 3), conforme Figura 4. 


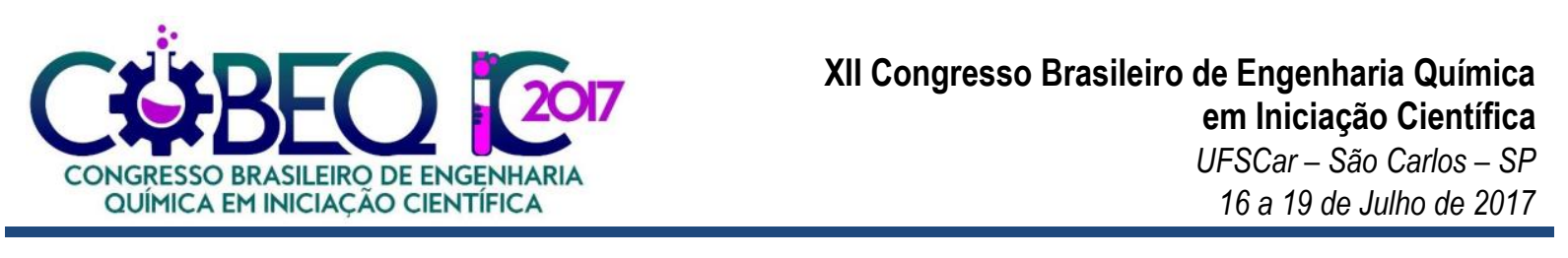

Figura 4 - Eficiência fracionária experimental e teórica (0,3 L/min de vazão de líquido).

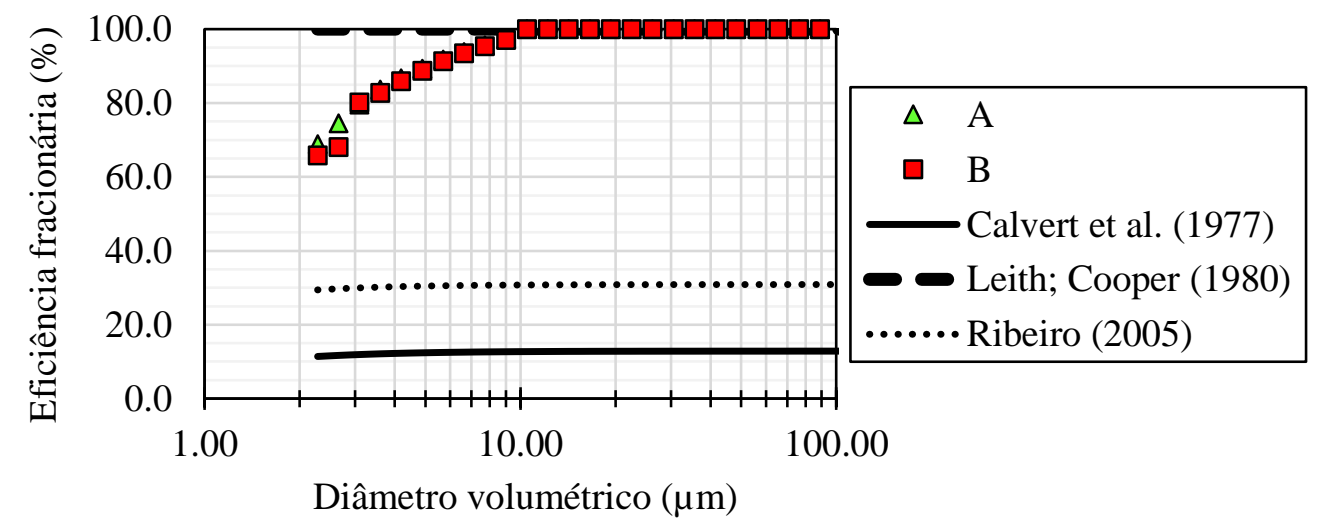

A Figura 4 permite observar que as equações de predição de eficiência não se ajustaram adequadamente aos dados experimentais para as condições operacionais utilizadas nos experimentos. A equação de Calvert et al. (1977), assim como a modificação desta realizada por Ribeiro (2005), subestimaram os dados experimentais, enquanto a equação de Leith e Cooper (1980) os superestimou na faixa abaixo de $10 \mu \mathrm{m}$, que era de interesse deste trabalho. Contudo, próximo a $8 \mu \mathrm{m}$, esta última conseguiu prescrever a totalidade de eficiência de coleta de particulado com o lavador Venturi para as condições operacionais empregadas neste estudo. Embora importante para o desenvolvimento da teoria sobre lavagem de gases com lavadores Venturi, a equação de Calvert et al. (1977) é uma modelagem do processo que considera exclusivamente o mecanismo de impactação inercial e relaciona parâmetros dos fluidos de trabalho e da partícula (inseridos no parâmetro $\Psi$ ). A modificação proposta por Ribeiro (2005), por sua vez, insere um parâmetro geométrico intrínseco ao equipamento e que considera o tempo de residência da partícula na garganta, embora seja uma correlação experimental e, portanto, foi sujeita a imprecisões. A equação de Leith e Cooper (1980) apresenta uma formulação teórica que engloba a queda de pressão oriunda do processo e a aerodinâmica da partícula, aspectos imprescindíveis para o entendimento dos fenômenos envolvidos no processo.

\section{CONCLUSÕES}

O aumento do diâmetro de particulado influenciou positivamente nos mecanismos de impactação inercial e interceptação direta e na eficiência do processo por consequência.

Para uma mesma vazão de gás, o aumento da vazão de líquido nas condições do experimento influenciou positivamente na coleta de partículas.

Dentre as equações da literatura utilizadas no trabalho, a correlação de Leith e Cooper (1980) melhor representou os dados experimentais.

\section{NOMENCLATURA}

\subsection{Siglas}

EPA - Environmental Protection Agency

$\mathrm{PM}_{10}$-Material particulado (Particulate Matter) de diâmetro menor que 10 micrômetros WHO - World Health Organization (Organização Mundial de Saúde) 


\subsection{Letras e Símbolos}

$C$ - Fator de Cunningham [adimensional]

$d$ - Diâmetro [L]

$\overline{D_{x}}$ - Diâmetro de Sauter da gota [L]

$f$ - Fator empírico da equação de eficiência de Calvert et al. (1977) [adimensional]

$l_{t}$ - Comprimento da garganta [L]

$Q$ - Vazão volumétrica $\left[\mathrm{L}^{3} \mathrm{~T}^{-1}\right]$

$u$ - Velocidade do fluido [ $\mathrm{L} \mathrm{T}^{-1}$ ]

$\beta$ - Fator empírico da equação de eficiência de Leith e Cooper (1980) [adimensional]

$\Delta p$ - Queda de pressão [M L $\left.\mathrm{M}^{-1} \mathrm{~T}^{-2}\right]$

$\eta$-Eficiência [adimensional]

$\mu$ - Viscosidade dinâmica do fluido $\left[\mathrm{M} \mathrm{L}^{-1} \mathrm{~T}^{-1}\right]$

$\rho$ - Densidade $\left[\mathrm{M} \mathrm{L}^{-3}\right]$

$\psi$ - Fator de impactação inercial [adimensional]

\subsection{Subíndices}

$g$ - Gás carreador

$l$ - Líquido de injeção

$p$ - Particulado

\section{REFERÊNCIAS BIBLIOGRÁFICAS}

CALVERT et al., 1977 apud COOPER, C. D.; ALLEY, F. C. Air pollution control: a design approach. 3rd ed. Long Grove: Waveland Press, 2002.

HESKETH, H. E. Air pollution control: traditional and hazardous pollutants. Lancaster: Technomic, 1996.

LEITH; COOPER, (1980) apud COSTA, M. A. M.; HENRIQUE, P. R.; GONÇALVES, J. A. S.; COURY, J.R. Droplet size in a rectangular Venturi scrubber. Brazilian Journal of Chemical Engineering. Vol. 21, n. 02, p. 335 - 343, abr.-jun. 2004.

RIBEIRO, A. P. R. A. Estudo da eficiência de coleta em um lavador Venturi com injeção de líquido por multi-orifícios. 2005. 93 f. Tese (Doutorado) - Departamento de Engenharia Química, Universidade Federal de São Carlos, São Carlos, 2005.

U.S. ENVIRONMENTAL PROTECTION AGENCY (EPA). Particulate Matter (PM) Pollution. Disponível em: https://www.epa.gov/pm-pollution. Acesso em: 23 ago. 2016.

WORLD HEALTH ORGANIZATION (WHO). WHO Air quality guidelines for particulate matter, ozone, nitrogen dioxide and sulfur dioxide. Global update 2005 - Summary of risk assessment. 2006. Disponível em: 〈http://www.who.int/phe/health_topics/outdoorair/outdoorair_aqg/en/>. Acesso em: 23 ago. 2016.

\section{AGRADECIMENTOS}

Os autores agradecem ao CNPq pelo auxílio financeiro. 